\title{
Designing and Constructing Log Periodic Dipole Antenna to Monitor Solar Radio Burst: e-Callisto Space Weather
}

\author{
Z. S. Hamidi, Z. A. Ibrahim, Z. Z. Abidin, M. F. Maulud, N. N. Radzin, N. Hamzan, N. M Anim, and
}

\author{
N. N. M. Shariff
}

\begin{abstract}
A Log Periodic Dipole Antenna (LPDA) for solar burst monitor in the range of $(45-870) \mathrm{MHz}$ applications is presented. In this paper, we make an effort in designing a portable LPDA that suitable with the criteria, specification and practical enough although is the boom length size of the antenna is 5.45 meter. We choose value of 0.14 spacing factor $\tau=0.8$ as a directivity of antenna. This LPDA is constructed by using two (2) rod aluminums type with nineteenth (19) elements of different sizes. Some of suggestions on improving this design are also presented.
\end{abstract}

Index Terms-Log Periodic Dipole Antenna (LPDA); ecallisto; solar burst.

\section{INTRODUCTION}

The Log Periodic Dipole Antenna (LPDA) is one of the broadband antennas that suitable for many applications. This coplanar linear array of unequal and unequally spaced parallel linear dipoles fed by a twisted balanced transmission line. With straightforward design introduced by Carrel, this is one of the advantages compared with other types of antenna. In our work, we design log periodic antenna is that fulfil the criteria to maximize the range of frequency from $45 \mathrm{MHz}$ till $870 \mathrm{MHz}$ The dimension of logperiodic antenna vary with frequency is the best type for this purpose because it's normally exhibit different radiation properties at different frequency and can be designed for any band, from High Frequency (HF) till Ultra High Frequency (UHF). The purposed of constructing LPDA is to monitor solar radio flux in due to solar flare phenomena. This is the standardized frequency range for e-CALLISTO networking. It also part of ISWI (International Space Weather Initiative) project in order to monitor the solar activity in radio region. There are few countries that participated and Callisto spectrometers have been deployed this research all over the world including Malaysia. We have just started this research in early 2011 with National Space Agency (ANGKASA) as the host and University Malaya and Universiti Teknologi MARA as a main university to enhance this project. The main objectives of this research is to monitor 24hours per day coverage of solar observations in state of support develop countries participate internationally with a latest technologies of instrument. Radio waves at meter and decimeter waves can be used as a diagnostics of the solar

Manuscript received March 9, 2012; revised April 20, 2012.

Z. S. Hamidi, M. F. Maulud, N. N. Radzin, and N. Hamzan are with Universiti Teknologi MARA, Shah Alam, Selangor, Malaysia (email: zetysh@salam.uitm.edu.my).

Z. A. Ibrahim, Z. Z. Abidin, N. M. Anim, N. N. M. Shariff are withUniversity Malaya, Kuala Lumpur, Malaysia. processes (review by McLean and Labrum 1985; theory review by Benz 2002). Scientifically, typical tasks to be solved in analyses of solar flares are: i) to identify active flare sources, ii) to discover their nature and emission mechanisms, and iii) to relate their properties with parameters of plasmas and accelerated particles in flaring regions. The instruments observe automatically, their data is collected every day via internet and stored in a central data base. As a part of worked, constructing antenna is the initial work that need to be done. We also have made some effort such as investigating the level of Radio Frequency Interference (RFI) level at National Space Centre at Banting, Selangor. This is important to fulfil the criteria and standard specification of the site and LPDA itself. Here we report on modification of constructing Log Periodic Dipole Antenna (LPDA).

\section{DESIGNING LPDA AND SOME IMPORTANT PARAMETER OF LPDA}

In this section, we discussed some important parameter and aspect that need to be considered. As we know, Log Periodic Dipole Antenna (LPDA) is frequency independent in that the electrical properties such as the mean resistance level $R_{0}$, characteristic impedance of the feed line $Z_{0}$ and driving-point admittance $Y_{0}$, vary periodically with the logarithm of the frequency. Here it the basic formula of determine the range of frequency due to elements. As the frequency $f_{1}$ is shifted to another frequency $f_{2}$ within the pass band of the antenna, the relationship is;

$$
f_{2}=f_{1} / \tau
$$

where, $\tau$ is a design parameter $(\tau<1.0)$

$$
L_{2}=\frac{\tau}{1} \text { and } L_{n}=\frac{\tau}{n-1}
$$

where

$f_{l}$ is lowest frequency

$f_{n}$ is highest frequency

$\tau$ is used to determining the element length $L$, and the element spacing $d$

$$
f_{3}=\frac{f_{1}}{\tau^{2}} \text { and } f_{n}=\frac{\tau}{n-1}
$$

where $d_{2-3}$ is spacing between elements 2 and 3. Table 1 showed the specification including the size of length and spacing between each element. 


$$
d_{2-3}=\tau d_{1-2}, d_{3-4}=\tau d_{2-3} \ldots d_{(n-1)-n}=\tau d_{(n-2)-(n-1)}
$$

where $d_{2-3}$ is spacing between elements 2 and 3. Table 1 showed the specification including the size of length and spacing between each element. Table 1 showed the specification including the size of length and spacing between each element.

TABLE I: SPECIFICATION OF 45-870 MHz LPDA

\begin{tabular}{cccc}
\hline No of elements & $\mathbf{L}(\mathbf{m})$ & $\mathbf{R}(\mathbf{m})$ & $\mathbf{D}(\mathbf{m})$ \\
\hline $\mathbf{1}$ & 3.96533329 & 5.551466963 & 1.110293326 \\
$\mathbf{2}$ & 3.17226668 & 4.441173636 & 0.888234674 \\
$\mathbf{3}$ & 2.53781338 & 3.552938962 & 0.710587750 \\
$\mathbf{4}$ & 2.03025073 & 2.842351212 & 0.568470208 \\
$\mathbf{5}$ & 1.62420061 & 2.273881003 & 0.454776173 \\
$\mathbf{6}$ & 1.29936050 & 1.819104830 & 0.363820944 \\
$\mathbf{7}$ & 1.03948842 & 1.455283885 & 0.291056759 \\
$\mathbf{8}$ & 0.83159075 & 1.164227125 & $0 . .232845411$ \\
$\mathbf{9}$ & 0.66527261 & 1.164227125 & 0.186276331 \\
$\mathbf{1 0}$ & 0.53221809 & 0.745105382 & 0.149021067 \\
$\mathbf{1 1}$ & 0.42577448 & 0.596084315 & 0.119216855 \\
$\mathbf{1 2}$ & 0.34061959 & 0.476867459 & 0.095373486 \\
$\mathbf{1 3}$ & 0.27249567 & 0.381493973 & 0.076298790 \\
$\mathbf{1 4}$ & 0.21799654 & 0.305195182 & 0.061039032 \\
$\mathbf{1 5}$ & 0.17439723 & 0.244156150 & 0.048831227 \\
$\mathbf{1 6}$ & 0.13951779 & 0.195324922 & 0.039064982 \\
$\mathbf{1 7}$ & 0.11161423 & 0.156259940 & 0.031251986 \\
$\mathbf{1 8}$ & 0.08929139 & 0.125007954 & 0.025001589 \\
$\mathbf{1 9}$ & 0.07143311 & 0.100006365 & 0.020001271 \\
\hline
\end{tabular}

\section{RESULTS AND DISCUSSIONS}

The antenna has a boom length $5.45 \mathrm{~m}$ which covered the range from $45 \mathrm{MHz}$ till $870 \mathrm{MHz}$ with gain $7 \mathrm{dBi}$. Due to principal of LPDA, the length, width and spacing of the nineteenth (19) elements of a log periodic antenna increases logarithmically and decreases smoothly, as one move away from the feed point. The lengths of elements are as high as 3.96 meters and as low as 0.071 meters. It is hope that this technique has focused on optimizing the accuracy and dynamic range achievable in the continuum burst by suppressing deconvolution errors that arise when the spectral structure of the sky-brightness is neglected. We also need to make sure that the low frequency-dependence of radiation pattern and input impedance. This can be done by make sure that all metallic parts are electrically connected to the mast flange and thus offer the high protection against electrostatic charges. By using two (2) boom lengths, we hope that the elements are balanced enough since the length is quite long which need a good front-to-back ratio of the antenna patterns of signal direction. However, we extend the axial length up to 6 meter for a handling the antenna purpose. In order to make a portable Log Periodic Dipole Antenna, the size of boom length becomes an issue. We divided into three of sizes, 2 meters respectively. We then need to make a hole for screw to joint it again.

\section{CONCLUSIONS}

It is important to have a proper method in order to fulfill a standard and accuracy measurement of log periodic dipole antenna (LPDA). This linearly polarized log-periodic antenna is the most practical transmitting antennas provide general broadband transmission and reception in wide range frequency. Although Log Periodic Dipole Antenna (LPDA) consists of a system of driven element, but not all elements in the system are active on a single frequency of operation. Due to different lengths and different relative spacing, it allowed changes in frequency to be made without greatly affected the electrical operation. We used the logarithmic periodic antenna directly connected via a low loss coaxial cable to the measuring instrument. The antenna is mounted horizontally on a steerable azimuth/elevation tower, and controlled by the computer to automatically point the sun during the day time. In order to make sure the log periodic antenna is high quality, aspect of material cannot be neglected. Therefore, we used the aluminium type a conductor material for elements and the PVC as an insulator of LPDA. These materials will against lighting strokes if happened.

The construction of log periodic dipole antenna (LPDA) has been presented. There is no doubt the log periodic dipole antenna is a best type of broadband applications so far. It will be setup at National Space Agency, Banting Selangor. To reduce noise, we also strongly recommend the installation of log periodic antenna as far as possible from radio frequency interference sources. Although there are still needs to be improved, this design of LPDA is our best construction so far. However, we realize that we need to develop the designing especially on how to maximize the gain and cable connection. Mounting aspect and tracking with the Sun also should be considered. Our next task is to test the performance of our LPDA and observe the output. We hope that we could gain the relevant data using this LPDA.

\section{ACKNOWLEDGEMENTS}

The author would like to thanks to University Malaya, Universiti Teknologi MARA, and National Agency Space of Malaysia (ANGKASA) for the collaborations of eCALLISTO. The development, production and distribution of CALLISTO spectrometers it supported by ETH engineer. The author also would like to thanks to C. Monstein from Switzerland and Prof Dr Zainol Abidin Ibrahim for the technical part of advice. This work was partially supported by the Excellence Fund UiTM and PPP UM P346/2010B grants. This project is an initiative work of the International of Space Weather Initiative (ISWI) program.

\section{REFERENCES}

[1] A. O. Benz, Christian Monstein and Hansueli Meyer CALLISTO, A New Concept for Solar Radio Spectrometers, Kluwer Academic Publishers, The Netherlands, 2004.

[2] G. H. Zhai, W. Hong, K. Wu, and Z. Q. Kuai, "Wideband substrate integrated printed log-periodic dipole array antenna," IET Microw. Antennas Propag., vol. 4, Iss. 7, pp. 899-905, 2010.

[3] M. S. M. Aras, M. K. A. Rahim, Z. Rasin, and M. Z. A. Abdul Aziz, "An Array of Dielectric Resonator Antenna for wireless application," 
IEEE International RF and Microwave Conference Proceedings, pp. 459-463, Dec 2008.

[4] R. Carrel, "The design of the log-periodic dipole antenna," IRE Int. Conv. Rec., 9, pp. 61-75, 1961

[5] G. Ia. Smolkov, A. A. Pistolkors, and T. A. Treskov, et al., Astrophys. Space Sci. vol. 119, no. 1, pp. 1, 1986.

[6] V. V. Grechnev, S. V. Lesovoi, G. Y. Smolkov, et al., Solar Phys. Vol. 261, no. 1-2, pp. 239, 2003.

[7] J. Carr, "Some variations in log-periodic antenna structures," IRE Trans. Antennas Propag., vol. 9, no. 2, pp. 229-230, 1961.
[8] A. O. Benz, Plasma Astrophysics, 2nd edn. (Springer, Dordrecht, 2002)

[9] N. Gopalswamy, "Radio Observations of Solar Eruptions, Solar Physics with the Nobeyama Radioheliograph," in Proceedings of Nobeyama Symposium, pp. 81-94, 2006.

[10] D. J. McLean and N. R. Labrum, Solar Radiophysics: Studies of Emission from the Sun at Metre Wavelengths (Cambridge University Press, Cambridge, 1985) 